\title{
Analysing the Media Streaming for QoS in Content Delivery Networks
}

\author{
Sachin Yadav \\ Research Scholar \\ Department of Computer Science \& \\ Engineering \\ UTU, Dehradun (INDIA)
}

\author{
Shailendra Mishra, PhD. \\ Professor \& HOD \\ Department of Computer Science \& \\ Engineering \\ KEC, Dwarahat (INDIA)
}

\author{
Ranjeeta Yadav \\ Assistant Professor \\ Department of Electronics \& \\ Communication Engineering \\ ABES, Ghaziabad (INDIA)
}

\begin{abstract}
The prediction of performance in CDN (Content Delivery Network) used to improve the technology development. The proposed analysis used how to design a network according to the applications of CDN network experiments with different workloads. This paper shows the variations of any parameter must affect the performance of content delivery network. The Average end-to-end delay, for Quality of service requirements, which are evaluated in this paper and event analysis also, be performed.
\end{abstract}

Keywords: Content Delivery Network, AODV, QOS, Routing Protocol.

\section{INTRODUCTION}

Content Delivery Network consists of many mobile nodes that act as both hosts as well as router in the free space air. Hence Content Delivery Network works without any pre-existing infrastructure. Since the quality of service is big challenge in the field of wireless Content Delivery Networks [1] [2] [3] [4]. Since mobile nodes are plug-n-play devices they have to face many issues in many applications of wireless communications. The field of wireless networking emerges from the integration of personal computing, cellular technology, and the Internet. This is due to the increasing interactions between communication and computing, which are changing information access from "anytime anywhere" into "all the time, everywhere." At present, a large variety of networks exist, ranging from the well-known infrastructure of cellular networks to non-infrastructure wireless ad hoc networks. When any link of a multi-hop communication path breaks, the path must be repaired or reconstructed. During this period, packets may be dropped. This loss of packets will affect quality of service (QoS) for both real-time and non-real-time applications and cause significant throughput degradation [5] [6]. So, we propose different workload with some parameter variations of the Content Delivery Network to evaluate performance metrics. There are different mobility models are available depends on mobility character. Based on Random way point mobility model, each node's movement consists of a sequence of random length intervals called mobility epochs during which a node moves in a constant direction at a constant speed [11] [12]. The speed and direction of each node varies randomly from epoch to epoch.

There are different types of mobility models. One is the entity mobility models and the other is the group mobility models. The random mobility model is the entity mobility model.

\section{PROTOCOLS}

There are different types of routing protocols are available, that is proactive, reactive and hybrid protocols. Reactive routing protocols for Content Delivery Networks are also called "ondemand" routing protocols. In a reactive routing protocol, routing paths are searched only when needed. A route discovery operation invokes a route-determination procedure. The discovery procedure terminates when either a route has been found or no route is available after examination for all route permutations. In a content delivery network, active routes may be disconnected due to node mobility. Therefore, route maintenance is an important operation of reactive routing protocols. Compared to the proactive routing protocols for content delivery networks, less control overhead is a distinct advantage of the reactive routing protocols. AODV, which is a reactive protocol.

\subsection{AODV}

The AODV routing protocol supports multi-hop routing among mobile nodes for establishing and maintaining an ad hoc network. AODV uses hop-by-hop routing. AODV is based on the Destination Sequence Distance Vector (DSDV) routing protocol [18]. It borrows the conception of sequence numbers from DSDV, plus the use of the on-demand mechanism of route discovery and route maintenance from DSR. It is called a "pure on-demand route acquisition system"; nodes that do not lie on active paths neither maintain any routing information nor participate in any periodic routing table exchanges. It is loopfree, self-starting, and scales to large numbers of mobile nodes.

\subsection{AODV ALGORITHM}

AODV operates on a series of request and responses to requests. Node constructing the route to another node sends to all the request (RREQ). The task includes the source and destination IP, the current time according to a source (13) and the time of last known connection, known by the source (17) and the request ID $(0 \mathrm{xFF})$. Each node receiving the request $0 x F F$ updates temporary routing table to the source, and:

2.2.1 If previously received a request from the source $0 \mathrm{xFF}$, ignores it.

2.2.2 If is the destination, or knows newer route (e.g. with time sends RREP to the source.

\subsubsection{If not, propagates the request.}

\subsection{AODV FEATURES}

As reactive routing protocol, AODV reacts relatively quickly to the topological changes in a network and updates only hosts that may be affected by the change. However, AODV tends to cause heavy overhead due to the flood search triggered by link failures. As a result, AODV does not perform well in heavy load or content delivery networks.

Main advantages are: 


\subsubsection{Algorithm is nor computationally or memory complex.}

\subsubsection{Data transfer does not generate additional traffic.}

\subsubsection{Scalable, suitable for mobile networks.}

2.3.4 Supports multicasting, tries to minimize the number of required broadcasts.

\subsection{CDN ARCHITECTURE}

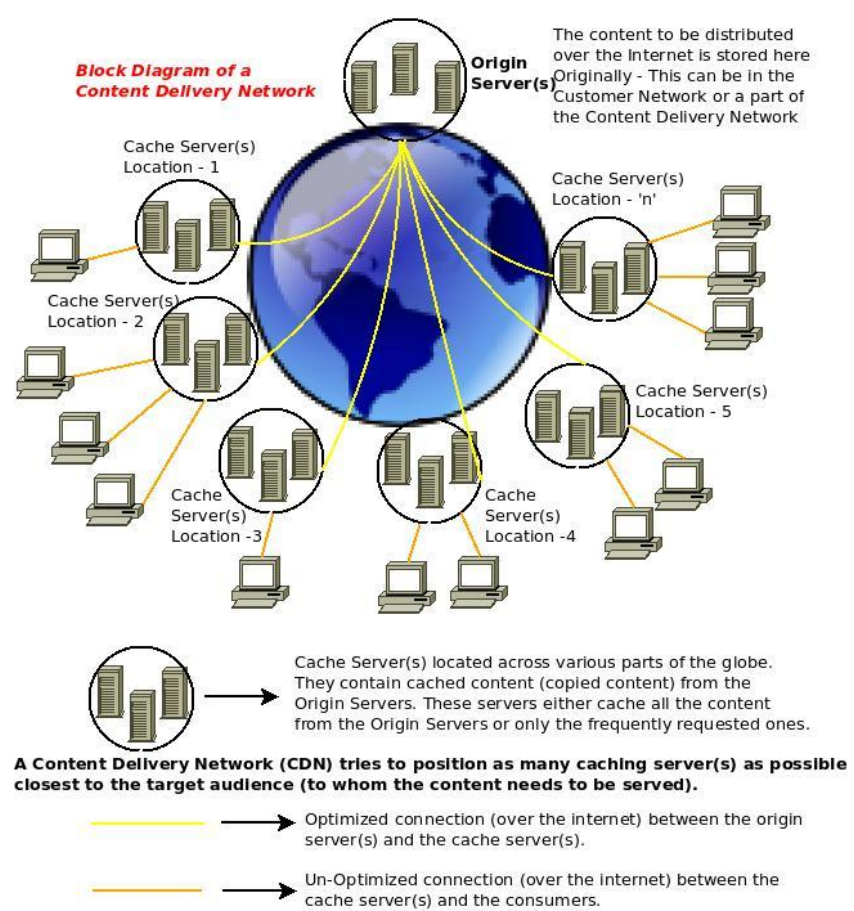

Figure 1: CDN Architecture diagram.

\section{METHODOLOGY}

This paper proposes the quality of service in AODV mobile adhoc networks. The data bytes are changed and the performance where evaluated throughout the networks. The data byte is changed as 500,1000, 1500 and 2000 respectively. This paper shows how the analysis Content Delivery Networks under these data rates changes. These performances are carried out for small, medium and large scale scenarios. The small scale scenario was 20 nodes, medium scale scenario 40 and 60 nodes and the large scale scenario 80 and 100 nodes. The simulation was repeated for perfect evaluation .

\section{PROBLEM STATEMENT}

CDN is a difficult task to define and scientific research work. Most important challenges in $\mathrm{CDN}$ are to providing quality of service in multimedia. QOS [11] is usually defined as a set of service requirements that needs to be met by the net- work While, transporting a packet stream from a source to its destination.

\section{PROPOSED WORK}

Quality of Service [12] refers to the capability of a network to provide better service to selected network traffic over various technologies. This approach is based on a new concept of AODV. Many approaches like DIFFSERV, INTSERV were applied to provide quality of service. The previous example is SHARP, Which have proactive and reactive process according to their size zones network.

\section{RESULT AND DISCUSSION}

We implemented CDN approaches within the Network Simulator ns-2 [14]. The topology was created using the GTITM topology generation tool with the transit-stub model, having 100 nodes. A video file of 1 minute duration, having a data-rate of $100 \mathrm{Kbit} / \mathrm{s}$ was selected for all the simulations.

The below figures show the comparison of proposed QOS with other protocols separately. Other recital metrics with different variations were also calculated and observed. By observing the below results this method delivers high performance compared with the protocols separately.

\section{CONLUSION AND FUTURE WORK}

These performance metrics are used to know how the characteristics of AODV protocol in CDNs differs in different parameters change scenarios. This proposal gives very high throughput and small delay in quality of service AODV protocol of Content Delivery network. Our work consists of conditional, link study and performance phase for the provision of quality of service metrics in CDN for large scale networks. By observing the below chart the new concept QOS delivers high packet delivery ratio. In future security analyze will be carried out to this Content Delivery Networks. 


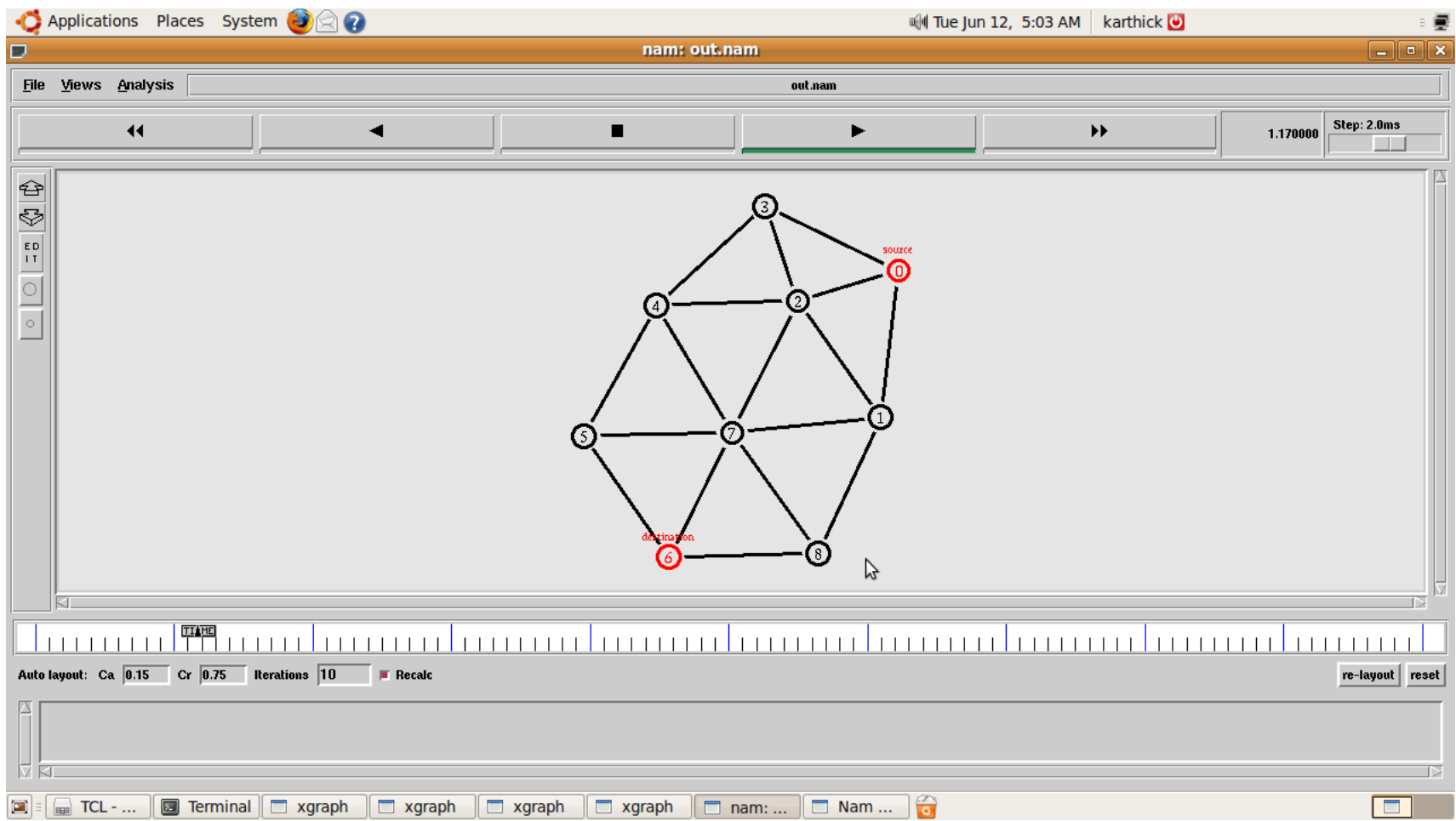

Figure 1. Network Topology(CDN).

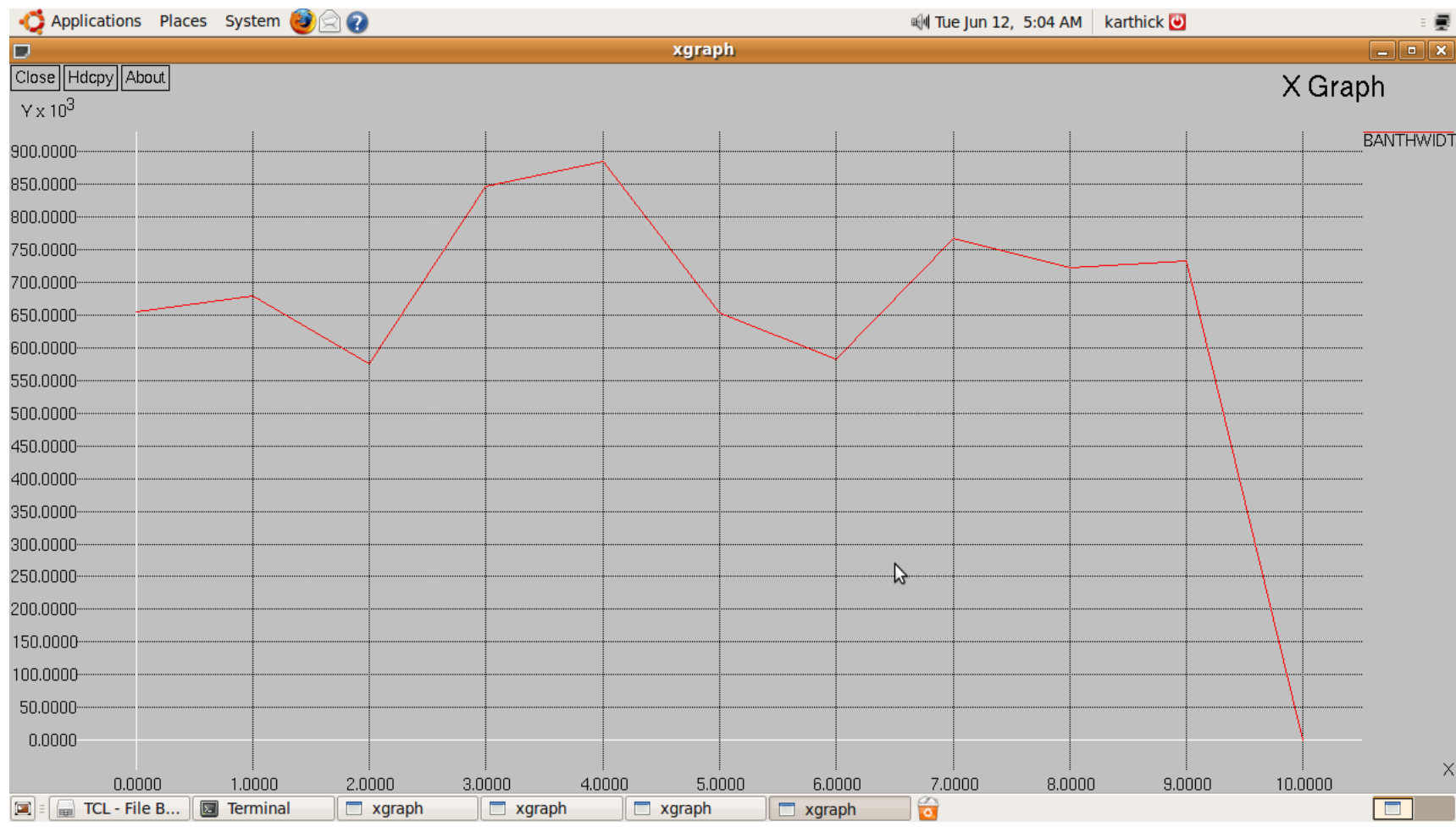

Figure 2. Bandwidth Utilization. 


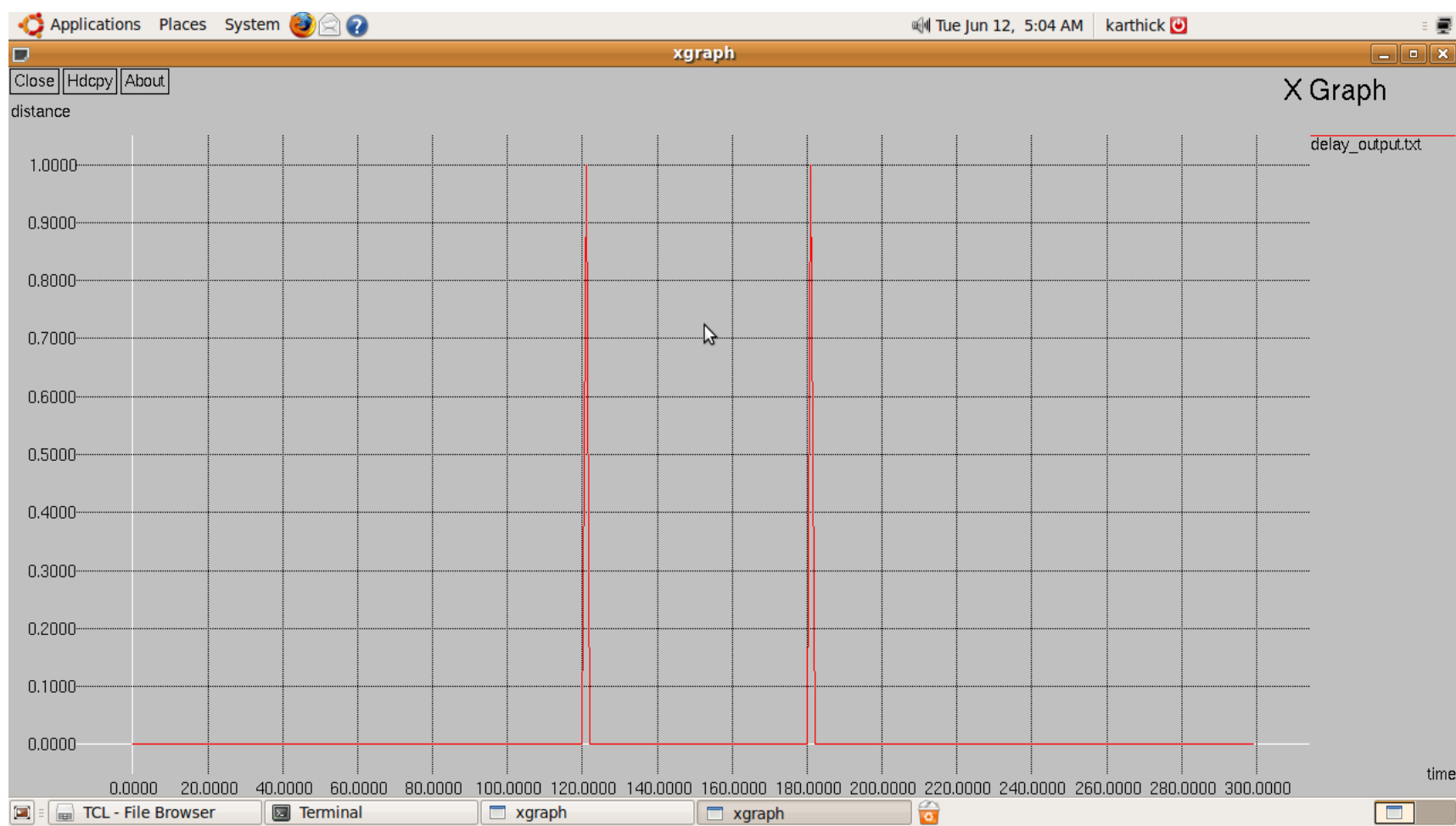

Figure 3. $\quad$ End To End Delay.

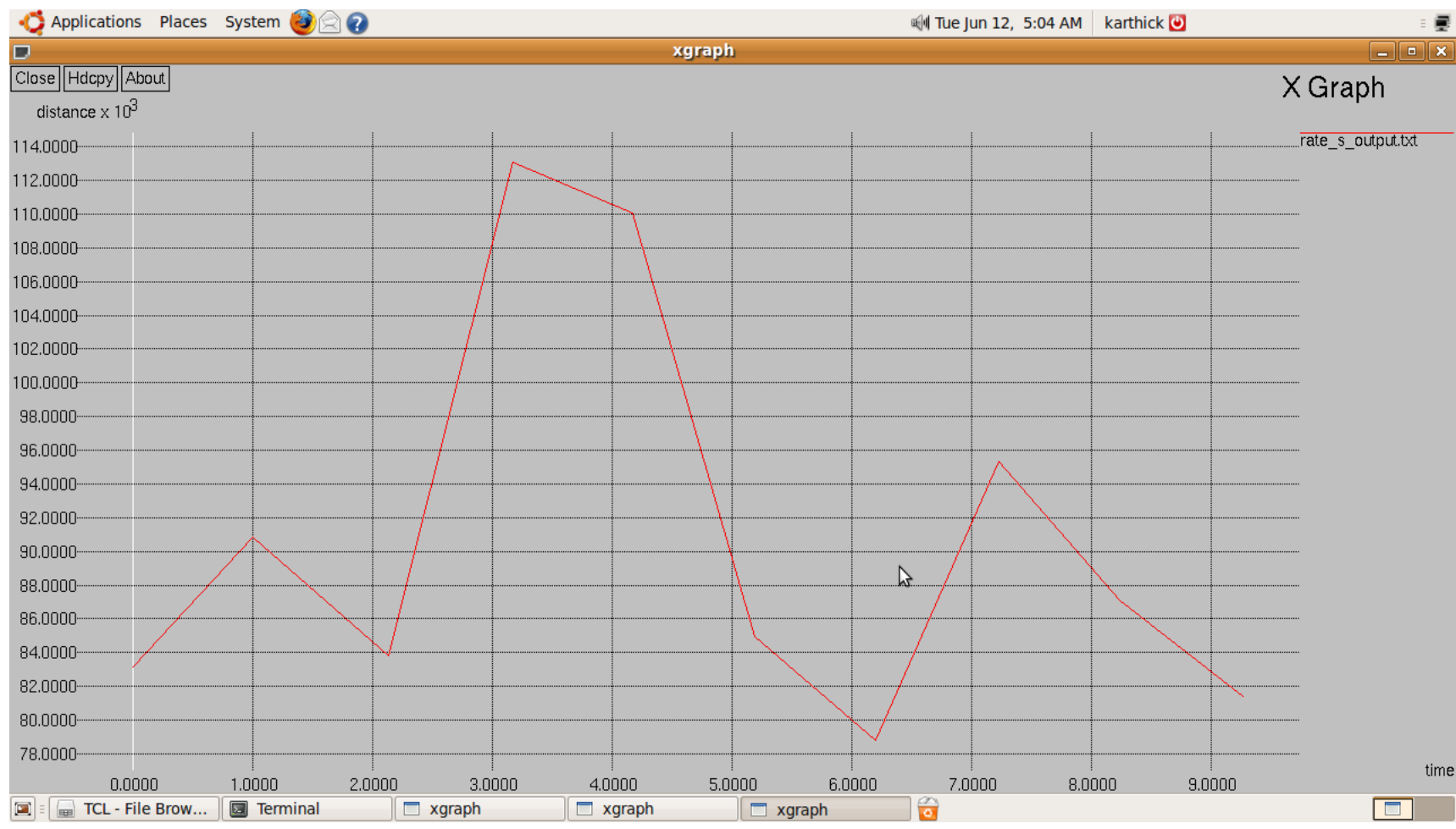

Figure 4. Error-Resilience For Sender. 


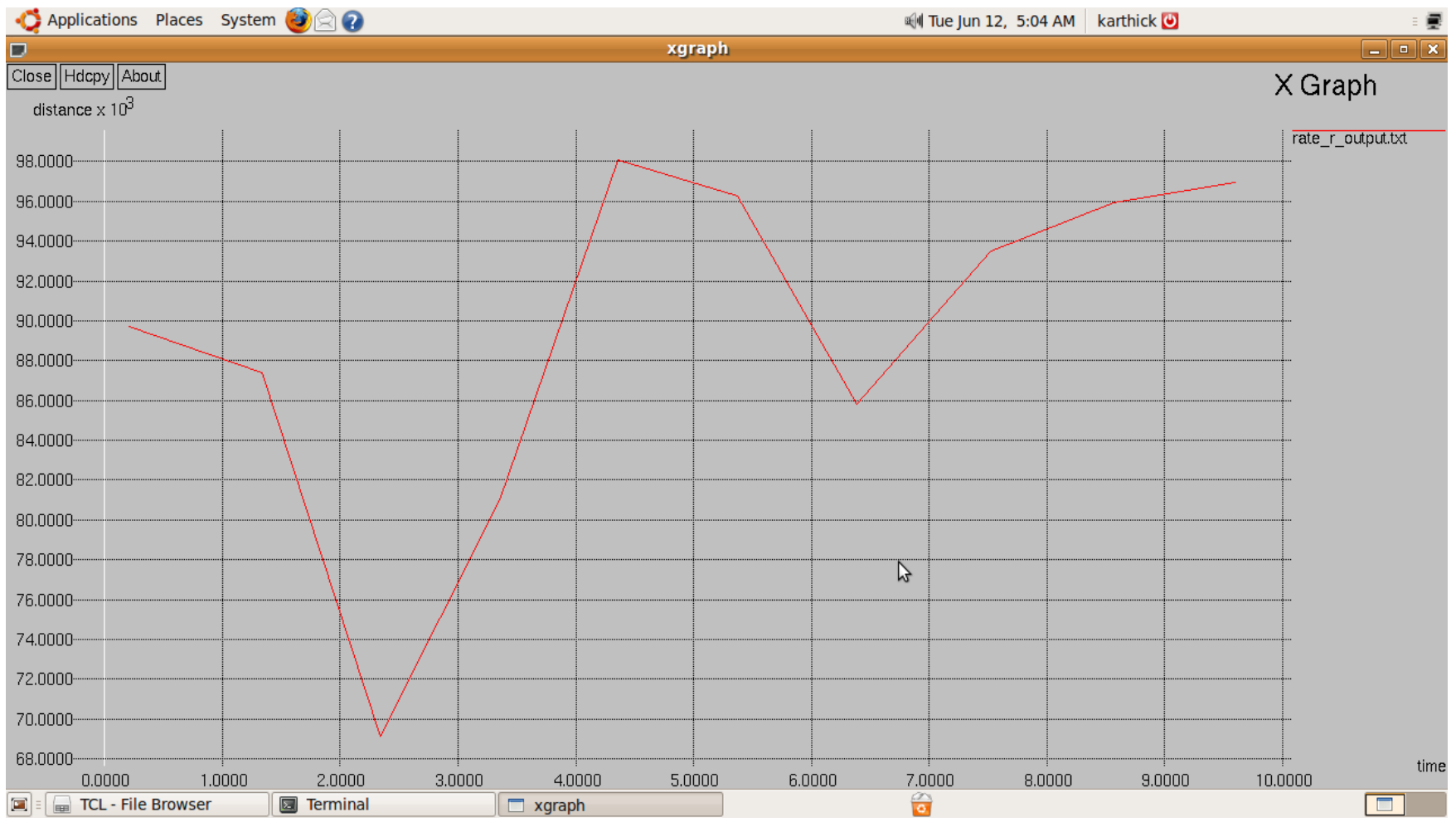

Figure 5.

Error-Resilience For Receiver.

\section{REFERENCES}

[1] P. Krishnan, D. Raz, and Y. Shavitt, "The Cache Location Problem," IEEE/ACM Transaction on Networking, Vol. 8, No. 5, 2000.

[2] L. Qiu, V. N. Padmanabhan, and G. M. Voelker, "On the Placement of Web Server Replicas," In Proceedings of IEEE INFOCOM, Anchorage, Alaska, USA pp. 15871596, April 2001.

[3] S. Jamin, C. Jin, A. R. Kure, D. Raz, and Y. Shavitt, "Constrained Mirror Placement on the Internet," In Proceedings of IEEE INFOCOM, Anchorage, Alaska, USA, April 2001.

[4] B. Li, M. J. Golin, G. F. Italiano, D. Xin, and K. Sohraby, "On the Optimal Placement of Web Proxies in the Internet," In Proceedings of IEEE INFOCOM, NY, USA, pp. 1282-1290, March 1999.

[5] Y. Chen, R. H. Katz, and J. D. Kubiatowicz, "Dynamic Replica Placement for Scalable Content Delivery," In Proceedings of International Workshop on Peer-to- Peer Systems (IPTPS 02), LNCS 2429, Springer-Verlag, pp. 306-318, 2002.

[6] Vakali, and G. Pallis, "Content Delivery Networks: Status and Trends," IEEE Internet Computing, IEEE Computer Society, pp. 68-74, November-December 2003.

[7] N. Fujita, Y. Ishikawa, A. Iwata, and R. Izmailov, "Coarse-grain Replica Management Strategies for Dynamic Replication of Web Contents," Computer Networks: The International Journal of Computer and Telecommunications Networking, Vol. 45, Issue 1, pp. 1934, May 2004.

[8] Y. Chen, L. Qiu, W. Chen, L. Nguyen, and R. H. Katz, "Efficient and Adaptive Web Replication using Content Clustering," IEEE Journal on Selected Areas in
Communications, Vol. 21, Issue 6, pp. 979-994, August 2003.

[9] G. Peng, "CDN: Content Distribution Network," Technical Report TR-125, Experimental Computer Systems Lab, Department of Computer Science, State University of New York, Stony Brook, NY, 2003.

[10] C. Yoshikawa, B. Chun, P. Eastham, A. Vahdat, T. Anderson, and D. Culler, "Using Smart Clients to Build Scalable Services," In Proceedings of USENIX.

[11] 3GPP technical Specification Group Services and System Aspects, "QoS Concept and Architecture", Release 1999, March 2000.

[12] R. Chowdhury, P. Bhandarker, M. Parashar, "Adaptive QoS Management for Collaboration in Heterogeneous Environments", Proc. IPDPS Heterogeneous Computing Workshop, pp. 234-255, Florida, April 2002.

[13] N. Semret, R. Liao, A.T. Campbell, A.A.Lazar, "Peering and Provisioning of Differentiated Internet Services", IEEE Infocom 2000,pp. 712-737, Tel Aviv, Israel, March 2000.

[14] The Network Simulator, http://www.isi.edu/nsnam/ns/

[15] J. Apostolopoulos, T. Wong, W. Tan, S. Wee, "On Multiple Description Streaming with Content Delivery Networks", IEEE INFOCOM, June 2002.

[16] V. K. Goyal. "Multiple Description Coding: Compression Meets the Network", IEEE Signal Processing Magazine, vol. 18, n.5, page(s) 74 - 94. September 2001.

[17] Braden R., Clark, D., and S. Shenker, "Integrated Services in the Internet Architecture: an Overview", Request for Comments, RFC-1633, 1994 